\title{
COMUNICACIÓN, SISTEMA E IMAGINARIO SOCIAL: LOS PUEBlOS MÁGICOS ANTE LA RESONANCIA DE LAS CRISIS ECONÓMICAS Y SANITARIAS
}

\author{
Milton ARAgón \\ miltonaragon@uadec.edu.mx \\ Universidad Autónoma de Coahuila \\ LUIS E. CASTRO \\ lucastros@uadec.edu.mx \\ Universidad Autónoma de Coahuila
}

La permanencia y pertinencia de los denominados Pueblos Mágicos para ciertos lugares del turismo se encuentra en entredicho, ante las crisis sanitaria y económica presentes. La raíz de ello radica en el proceso de construcción de sentido a partir del imaginario social. Se observa que los sistemas sociales comunicativos operacionalmente clausurados, poseen resonancia ante la crisis, solo cuando dicha resonancia se construye como operaciones dicotómicas internas, de ahí lo endeble de un denominador cuyo sentido fue externa, artificial y cosméticamente inducido desde políticas públicas con fines de explotación de valores de cambio en torno al turismo.

Palabras claves: Sistemas comunicativos, imaginarios sociales, turismo, Pueblos Mágicos.

\section{COMMUNICATION, SYSTEM AND SOCIAL IMAGINARY: the Pueblos MÁgicos In the face of the RESONANCE OF ECONOMIC AND HEALTH CRISES}

The permanence and relevance of the so-called Pueblos Mágicos for certain places of tourism is in question, mainly due to the current health and economic crisis. The root of this order of things is found in the process of construction of meaning from the social imaginary. It is seen that operationally closed communicative social systems, have resonance in the face of the crisis only when resonance is constructed as internal dichotomous operations, hence the weakness of a denominator whose meaning was external, artificial and cosmetically induced from public policies for the exploitation of exchange values around tourism.

Keywords: Communication systems, social imaginaries, tourism, Pueblos Mágicos.

Fecha de recepción: 11 de octubre de 2020

Fecha de aceptación: 16 de enero de 2021

CÓMO CITAR: Aragón, M. y Castro, L. (2021). Comunicación, sistema e imaginario social: los Pueblos Mágicos ante la resonancia de las crisis económicas y sanitarias. Dimensiones Turísticas, 5(8), 87-104. https://doi.org/10.47557/PCRU7660 


\section{Introducción}

L

os Pueblos Mágicos (PM) son una política pública, o mejor dicho fueron, ya que actualmente se encuentran como el gato de Schrödinger: se tiene el nombramiento mas no el recurso financiero, lo que los mantiene en un estado de incertidumbre, pues su objetivo como programa de gobierno es incentivar el turismo regional. Eso queda claro, como política pública se presenta además otra discusión sobre cómo opera la gestión del programa, dónde se evalúa y si sus resultados fueron los esperados para ser considerada una iniciativa gubernamental exitosa. Para este último punto se puede revisar el texto de Velázquez-García y Lara-Figueroa (2017), donde se estudian diferentes lugares del turismo en México desde las políticas públicas.

En la actualidad, los PM vienen arrastrando una crisis económica que se agudizó con la crisis sanitaria producto del COVID-19, lo cual lleva a reflexionar sobre cómo esos dos aspectos pueden traer cambios comunicativos desde la contingencia en los lugares del turismo en general y los PM en particular, por medio de la resonancia que aumenta precisamente el sentido de contingencia en la comunicación del sistema, poniendo en conflicto su mantenimiento. No obstante, el imaginario social puede delimitar la resonancia al introducir códigos comunicativos que posibiliten mantener el sentido desde el cual se produce la realidad (Aragón, 2017) como lugar de turismo en los PM.

De tal forma, el fenómeno de la producción de realidad en el contexto de la crisis económica y sanitaria en los PM se puede definir y desarrollar desde dos vertientes: 1) como construcción de la realidad por el acoplamiento de la comunicación y 2) como construcción de la realidad por medio de los imaginarios. La primera fue propuesta por Niklas Luhmann (1996a), donde presenta dicha construcción de la realidad como emergencia de los símbolos socialmente generalizados y la clausura operacional del sistema. La segunda fue establecida por Juan Luis Pintos a mediados de los 90 y hasta el 2018, quien retomó parte de los postulados teóricos-analíticos de Luhmann (1996a) y los llevó al estudio de los imaginarios sociales (Pintos, $2005 ; 2014 ; 2016)$.

Ambas propuestas mencionan que los códigos solo operan en el contexto comunicativo que se construye con la información del sistema, desde el cual emerge la realidad que mantiene el sentido de este. Su importancia radica en la entrada de 
códigos externos al sentido del sistema que lo transforman. De ahí que el sistema se clausure en su operar.

Lo interesante es que el turismo forma parte del sistema económico, pero con interpenetración del sistema social por medio de la resonancia que producen los imaginarios sociales sobre estos, pues ellos dotan de un sentido de recreación a una actividad económica. De tal manera, es parte del conflicto el querer conceptualizarlo como un sistema cultural, con el cual comparte códigos, pero su sentido va en función de mantener el sistema económico. De ahí que el objetivo del presente texto sea el reflexionar sobre cómo por medio de la resonancia se pueden reintroducir códigos al subsistema turístico, en función de las necesidades de los habitantes y no del sistema económico o político.

\section{Nota metodológica}

Como se menciona en el objetivo, lo que se busca es reflexionar por medio de una explicación desde la teoría de sistemas de segunda generación, un fenómeno que se está presentando en los PM. Por lo tanto, sobra decir que esto conlleva analizar los textos de Luhmann (1996a; 1996b; 2002; 2007; 2020) que puedan aportar a dicha explicación.

Cabe señalar que la técnica empleada es la hermenéutica analógica (Beuchot, 2009), con la cual se busca el vínculo entre lo que se ha observado en campo en diferentes trabajos anteriores (Aragón, 2015; Aragón et al., 2017a; Aragón et al., 2017b; Aragón et al., 2016; Aragón et al., 2014; Velázquez-García y Aragón, 2015) y la actual coyuntura por la cual pasan dichos lugares del turismo y sus implicaciones en la construcción de la realidad desde los imaginarios, conteniendo un excurso matemático que pretende denotar, por analogía, lo endógeno de la resonancia posible de un sistema. Es por ello que este trabajo es una investigación con inspiración en la teoría, en el sentido de Luhmann (2020, pp. 10-11), pues para él:

Las investigaciones que encuentran su inspiración en la teoría siempre pueden ser acusadas de una falta de "referencia práctica". No proporcionan prescripciones a otros. Observan la práctica y en ocasiones preguntan qué se gana con el uso apresurado de ideas incompletas. Esto no excluye la posibilidad de que procediendo así puedan alcanzarse resultados útiles. Pero entonces la importancia de la teoría siempre permanecerá en el sentido de que un método más contro- 
lado de producir ideas pueda aumentar la probabilidad de resultados más útiles $-\mathrm{y}$, sobre todo, de reducir la probabilidad de crear entusiasmo inútil-.

\section{La construcción de la realidad por el acoplamiento comunicativo}

En la construcción de la realidad por medio del acoplamiento comunicativo hay que tener claro que el operar de los sistemas comunicativos es autorreferencial y clausurado operativamente, características que permiten una construcción de la realidad a partir del sentido del turismo en el sistema económico.

El lenguaje, al carecer de un instrumento de observación que permita designar una realidad independiente como la operación en Luhmann (1996a), hace que no se constituya como un sistema. Para que se genere como un sistema necesita la unión selectiva de operaciones recursivas que permiten la autopoiesis por medio de la clausura de operaciones; pero como el lenguaje no tiene un operar autónomo, parte de una heterorreferencia determinada por el grupo lingüístico al cual pertenece, por lo tanto, es alopoiético (Aragón, 2011).

Si no operara de esta forma se complicaría la comunicación en el sistema social, pues el lenguaje construiría sus propias reglas para mantener su autopoiesis, presentándose una constante transformación de los signos lingüísticos y, por ende, de los códigos comunicativos, lo que dificultaría el acoplamiento y su simbolización socialmente generalizada, porque:

El lenguaje no posee ningún modo propio de operar, y no debe ser manejado como el acto mismo de pensar o como el de participar en la comunicación. Consecuentemente, el lenguaje no constituye un sistema propio. Es y seguirá siendo dependiente del hecho de que los sistemas de conciencia, por una parte, y el sistema de comunicación, por otra, prosigan la propia autopoiesis mediante operaciones propias completamente clausuradas. Si esto no sucediera, cesaría inmediatamente todo lenguaje, y luego toda posibilidad de pensar lingüísticamente. (Luhmann, 2002, p. 291).

El lenguaje es simbólicamente generalizado a través de los diferentes acoplamientos comunicativos que llevan a cabo los sistemas de conciencia en su comunicar. Estos sistemas de conciencia van a determinar la generación de la comunicación a 
partir de elementos del lenguaje, por medio de la autorreferencia y la heterorreferencia, en la configuración de la distinción con el sistema social.

De tal forma que "el lenguaje no es ningún sistema, porque no posee una forma específica de operación. La operación del lenguaje le adviene por la comunicación o por una realización de conciencia que introduce en el lenguaje las formas de sentido" (Luhmann, 2002, p. 294). Entonces, el lenguaje se presenta como un elemento fundamental en la estructuración de la información que compone el sistema comunicativo, el cual a su vez interpenetra en distintos sistemas por medio de signos autorreferidos a las comunicaciones de cada sistema acoplados recursivamente.

El lenguaje, al estar constituido por signos autorreferenciados en la operación comunicativa, es la base de la diferenciación y distinción llevada a cabo por el sistema comunicativo en su acoplamiento estructural, emergiendo la realidad como resultado de la construcción de estas operaciones de observaciones articuladas y determinadas estructuralmente, que son de manera simbólica generalizadas por los sistemas de conciencia.

De ahí que el carácter sistémico de la comunicación dependa de la forma en que es acoplado en el proceso de diferenciación entre designado/no designado, generando una paradoja que constituye la realidad como una unidad de la diferencia operacional de la observación, generándose autodescriptores que asignan un nombre a lo diferenciado. Porque es través del lenguaje que los sujetos establecen códigos de diferenciación con el entorno.

Esto se hace a través de procesos de diferenciación que son los que van a permitir marcar un ámbito en el que cada sistema funciona. Por ello, la realidad construida será aquello que es designado en función de las características de los códigos socialmente generalizados y acoplados por el sentido del sistema, pero esa realidad construida podría cambiar profundamente cuando se vea modificado el sistema de diferenciación, como el caso de la crisis, pues esta produce en el sistema resonancia cuando:

Los acontecimientos que suceden en el entorno solo pueden producir efectos en un sistema bajo condiciones muy especiales y sobre todo si se colocan en la propia frecuencia del sistema [que] conducen a una secuencia de reacciones en el sistema dependiendo de las condiciones estructurales del propio siste- 
ma. La resonancia, por tanto, es siempre resonancia delimitada, resonancia dependiente de las estructuras. (Luhmann, 1996b, p. 84).

Por ello la importancia de la diferenciación comunicativa del sistema que delimita a la resonancia y filtra los códigos para mantener el sentido del mismo, porque "si este no pudiera filtrar, sino que ante cualquier acontecimiento del medio ambiente pudiera quedar afectado, entonces no sería ningún sistema" (Luhmann, 1996b, p. 84). Al interior de su operación, el sistema emplea conceptos que se han designado y determinado por medio de un relativismo histórico, que le mantienen una coherencia estructural respecto al sentido.

Dichos conceptos con el tiempo se afianzan o desechan, con la finalidad de que el sistema pueda autodefinirse por medio del uso de contrastes. En su mayoría son dicotómicos y refieren a la alteridad, de ahí que sean usados para realizar la distinción entre los elementos del entorno por medio de códigos autodescriptores, los cuales confieren:

Un nombre, una designación rígida e invariante que en razón de esa rigidez siempre se repite y se puede aplicar en innumerables situaciones distintas e imprevisibles. Sobre estos nombres propios pueden apoyarse luego las contraposiciones que oponen al sistema otro sistema para identificarlo en el contraste [Esto permite] un paulatino ir llenando los contrastes con designaciones estructurales [...] y, con ello, un enriquecimiento del contenido del texto con el cual el sistema se autodesigna. A tales textos, incluyendo nombres, los llamaremos autodescriptores. (Luhmann, 2007, p. 698).

De ahí que los autodescriptores son componentes autorreferenciales que se designan como una autoindicación y una identidad basada en las diferencias/oposiciones, no en las similitudes otorgadas por sus propios elementos, los cuales refuerzan la diferenciación por medio del uso de signos significados por las convenciones sociales, que no son otra cosa más que los códigos con los que opera su acoplamiento el sistema comunicativo.

Los autodescriptores son una designación rígida e invariante que siempre se repite, operando como productores de códigos designados estructuralmente, lo que hace que se mantenga el sentido. En el caso del subsistema turístico como parte del sistema económico, esos autodescriptores corresponden a los textos derivados del 
valor de uso simbólico del patrimonio material, natural e inmaterial, codificado a partir de las necesidades estructurales del sistema económico.

De ahí que el sentido de los lugares turísticos sea en función del valor de uso, el cual ante las crisis se vuelve contingente, pues afecta el flujo de visitantes y, por lo tanto, el sentido del subsistema desde el código del valor de uso patrimonial puede llegar a transformarse perdiéndose la identidad del sistema, que en este caso sería que se abandonara la vocación turística de los PM, al no poder delimitar los códigos de la resonancia producto de la crisis económica y sanitaria.

Lo anterior se posibilitaría pues en la mayoría de los PM sus autodescriptores no provienen de un devenir histórico-cultural del lugar, sino impuestos desde las necesidades políticas para promover programas que incentivaran la economía por medio del turismo; de tal forma que sus códigos no llegan a ser designados estructuralmente, por lo cual se comportan más como un sistema alopoiético en los casos donde fue impuesto el PPM en zonas que carecían de elementos suficientes para sustentar un valor de uso patrimonial, pues los PM se codifican desde un discurso oficial externo que no parte del sentido de los sistemas de conciencia y la comunidad:

La marca nacional Pueblo Mágico se ha sobrepuesto a las marcas locales. La diversidad del mosaico cultural y geográfico ha dejado paso a las intervenciones en el cuadro central genérico, donde son reiterativos los usos del suelo de los principales hoteles y restaurantes y algunas franquicias. La cubierta general de concreto en pavimentos y banquetas ha intentado infructuosamente emparejar a los pueblos que, gracias a la defensa de ciertas permanencias y a la riqueza de recursos patrimoniales, mantienen su singularidad. De manera que la valoración promovida oficialmente no se basa tanto en los atractivos e intereses locales como en formas establecidas de intervención. (Méndez, 2017, p. 206).

El principal problema de que sus autodescriptores no representen los códigos con los cuales se quiere que opere el subsistema económico en los PM, se da justo en el exceso de contingencia al cual están expuestos con cualquier alteración en el entorno, como serían los cambios políticos y las crisis económicas y ambientales. Por ejemplo, en el caso del sistema político sus códigos van en referencia del éxito de los PM como un programa de política pública, sin importar si opera con el sentido del sistema económico, ya que introduce falsos códigos por medio de incentivos financieros los cuales en la actualidad han sido eliminados, creando una aguda 
crisis económica. Con ello, habría que reintroducir lo códigos que provienen de los sistemas de conciencia para delimitar la comunicación de la resonancia.

Aquí es donde planteamos que el imaginario social podría ser un código que genere un efecto positivo en los subsistemas políticos, que reoriente el sentido en función de sus propios códigos y necesidades no tanto de las necesidades operacionales del sistema político o económico, con lo cual podrían hacer frente a contingencias como las crisis, desde una clausura basada en el mantenimiento de la comunidad y no de elementos externos como son los políticos o los extranjeros.

\section{Excurso analógico: algunas precisiones matemáticas sobre el operar de la resonancia}

La resonancia en sistemas isomorfos (mecánicos, acústicos, ópticos, electrónicos, hidráulicos, etcétera) puede modelizarse matemáticamente en forma simple como un sistema dinámico lineal, con parámetros invariantes en el tiempo y con un solo grado de libertad; vale decir: un sistema vibratorio observado a lo largo del tiempo en un solo eje.

La representación simbólica de, por poner un ejemplo típico, las vibraciones de un sistema "resorte-masa-amortiguador con volante" (ver figura 1) en un rango pequeño de oscilaciones, es una ecuación diferencial lineal de segundo orden con coeficientes constantes, forzada por un senoide de frecuencia $\omega$, correspondiente a un balance de fuerzas (expresión 1) alrededor del centroide de masa del sistema en equilibrio de cuerpo libre, de acuerdo con la segunda ley de Newton:

$$
m \frac{d^{2} x}{d t^{2}}=-\beta \frac{d x}{d t}-k x+F o \operatorname{sen} \omega t
$$

En donde $x(t)$ es la posición del centroide en tiempo $t, d x / d t$ la velocidad instantánea en tiempo $t, d^{2} x / d t^{2}$ la aceleración instantánea en tiempo $t, m$ la masa total del sistema, $\beta$ la constante de amortiguamiento cinético (proporcional a la velocidad del sistema), $k$ la constante de restitución elástica del resorte (proporcional a la deformación pequeña, ley de Hooke), Fo (=mao) la fuerza del volante aplicada sobre el sistema con frecuencia $\omega$, en la dirección $x$. 
Figura 1. Sistema masa-resorte-amortiguador con volante

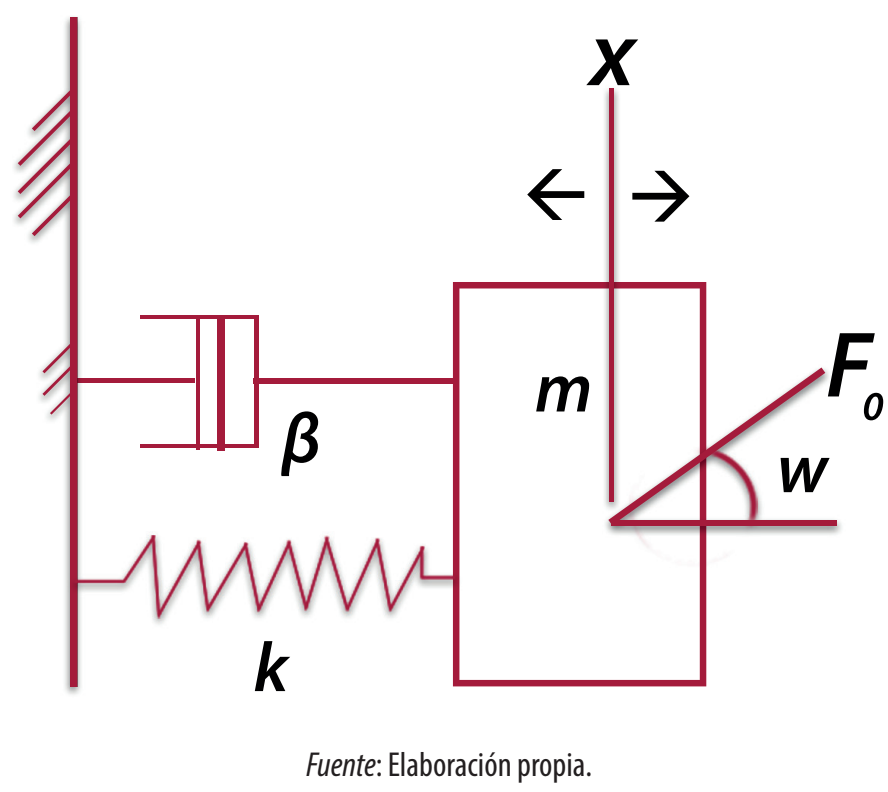

La ecuación (1) puede normalizarse por unidad de masa total del sistema, simplificando parámetros y reacomodando los términos para denotar lo interno (lado izquierdo) y lo externo (lado derecho) del sistema:

$$
\text { (2) } \quad\left\{\frac{d^{2} x}{d t^{2}}+\xi \frac{d x}{d t}+\omega_{0}^{2} x\right\}_{\text {lo interno }}=\left\{a_{0} \text { sen } \omega t\right\}_{\text {lo externo }}
$$

En donde $\xi$ es el factor de amortiguamiento $(\beta / \mathrm{m})$, $\omega 02$ es el cuadrado de la frecuencia natural de vibración $(\mathrm{k} / \mathrm{m})$ y a0 la aceleración del volante (Fo/m).

La solución analítica de la ecuación diferencial (3), proporciona una descripción de las vibraciones (oscilaciones del sistema en torno a su punto de equilibrio en el eje $\mathrm{X}$ ) a lo largo del tiempo. Se simplifica, sin pérdida de generalidad, representando el modelo en ausencia de amortiguamiento, que se reduce a:

(3) $\frac{d^{2} x}{d t^{2}}+\omega_{0}^{2} x=a_{0} \operatorname{sen} \omega t$

Con solución:

(4) $\quad x(t)=\left(\frac{a_{0}}{\omega_{0}^{2}-\omega^{2}}\right) \operatorname{sen} \omega t$ 
En donde la "amplitud" o factor de ganancia del sistema, A, es

$$
\text { (5) } \quad A=\frac{a_{0}}{\omega_{0}^{2}-\omega^{2}}
$$

Que muestra que A crece sin cotas si $\omega_{0}^{2} \rightarrow \omega^{2}$ (ver figura 2) y, por lo tanto:

$$
\lim _{\omega_{0}^{2} \rightarrow \omega^{2}} x(t) \rightarrow \infty
$$

Figura 2. Sistema vibratorio en resonancia.

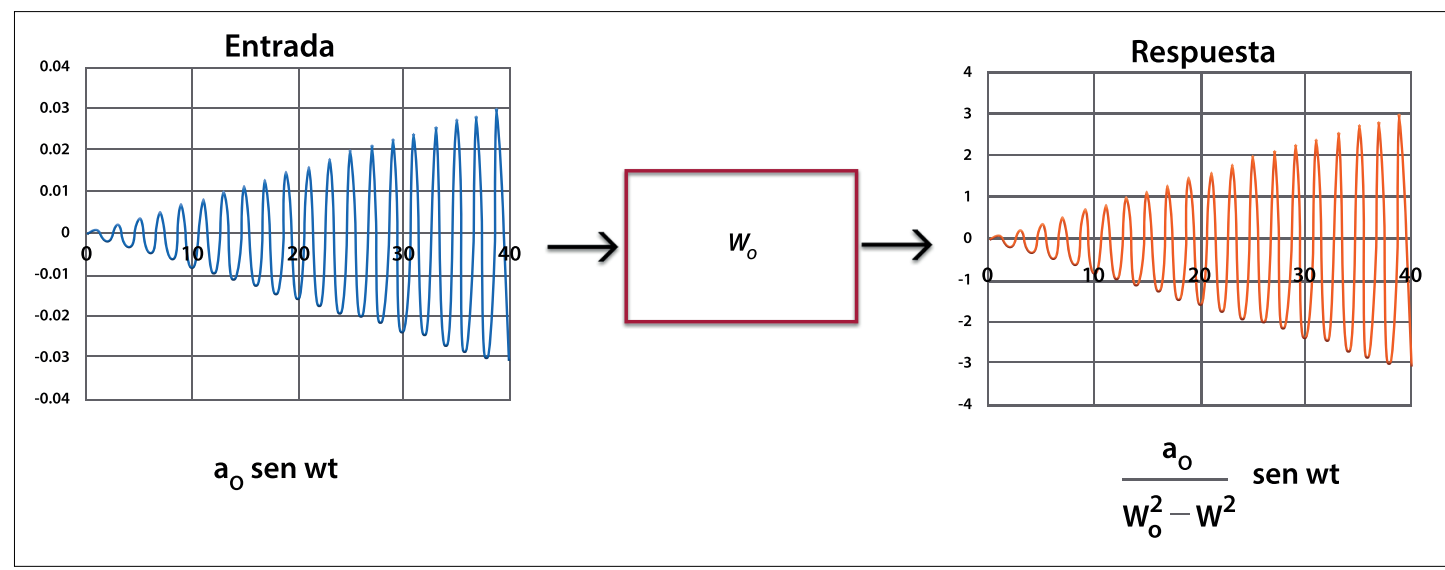

*La respuesta amplifica la fuerza externa cuando $\omega_{0}^{2} \rightarrow \omega^{2}$ Fuente: Elaboración propia.

La formulación para casos más generales es algo más elaborada, pero esencialmente se llega a las mismas conclusiones: el sistema entra en resonancia si la frecuencia natural de vibración coincide o es cercana a la frecuencia de la fuerza externa sobre el sistema; el sistema amplifica o responde con mayor intensidad a influencias cuya frecuencia se encuentra cerca de su propia frecuencia natural de vibración.

En la práctica, en una especie de proceso dialéctico natural el sistema sufrirá cambios cualitativos antes de alcanzar oscilaciones extremas; por ejemplo, se destruiría o deformaría por efecto de la vibración. La resonancia aparece como una propiedad del sistema, no como una propiedad de las fuerzas externas al sistema, pero sí como respuesta característica a estas, en donde ciertamente el sistema nota lo que está pasando "afuera". 
Mutatis mutandis, trasladándonos al reino de los sistemas sociales comunicativos operacionalmente clausurados, es decir, aquellos que solo se producen mediante códigos binarios de comunicación que producen más comunicación (por ejemplo, el de relevancia/opacidad que se verá respecto a los imaginarios) estructuralmente restringidos, se observará que desde este contexto la ecuación (2) aparece como una expresión para el acoplamiento estructural.

Los grados de libertad del sistema o número de direcciones en que se contempla la dinámica del sistema aparecen como las diferencias que dan lugar a las lógicas booleanas genéticas del mismo, en tanto que la frecuencia natural del sistema $(\omega)$ se muestra como el factor autopoiético del sentido, en el contexto de cada lógica binaria que da lugar a la respuesta estructural del sistema clausurado operacionalmente.

Por lo tanto, la ganancia (5) se ve como la capacidad de la irritación estructural para generar una respuesta. Por último, llevando la analogía al tope, la resonancia (6) se observa como la capacidad estructural del sistema para sentir irritaciones y expresar, siempre estructuralmente, cambios cualitativos de comportamiento en relación con el factor irritante en sistemas interpenetrados.

\section{La construcción de la realidad por medio de los imaginarios}

¿Es posible que las significaciones de los imaginarios sociales puedan producir códigos que delimiten la resonancia y la aprovechen al ser una propiedad comunicativa del sistema, para transformarlo en un sentido desde las necesidades de la comunidad? Pues de los imaginarios sociales se pueden producir códigos de reentrada vinculados al sentido de los sistemas de conciencia, estructurados a partir de una identidad como comunidad. Por lo tanto, operarían como autodescriptores originados en su deriva histórico-cultural designando estructuralmente a sus necesidades de acoplamiento como sistema y no a las de otros sistemas.

Porque los imaginarios sociales definidos desde las diferenciaciones funcionales de Luhmann, para Pintos (2005, p. 42) son "1. Esquemas socialmente construidos, 2. Que nos permiten percibir, explicar e intervenir, 3. En lo que cada sistema social diferenciado, se tenga por realidad". Precisando que los esquemas socialmente construidos hacen referencia al elevado grado de abstracción que operan, al igual que las referencias temporales que implican secuencias, prioridades y jerarquías de 
las percepciones por medio de la distinción socialmente diferenciada relevancia/ opacidad (Pintos, 2005). Esto es interesante porque marca la diferenciación entre lo asignado y no asignado, entre lo que es observado y acoplado y lo que no, permitiendo generar códigos que doten de sentido a la clausura operacional o delimiten la resonancia.

En lo que respecta a los verbos percibir, explicar e intervenir, para Pintos (2005, p. 43) "las operaciones complejas a las que se refieren estos verbos son posibles para nosotros porque disponemos de un 'mundo a nuestro alcance' y una distribución diferenciada del conocimiento [...] que posibilita unas referencias semejantes de percepción [...] de explicación [...] y de intervención". De ahí que los imaginarios incentiven una acción en cuanto al poder percibir, explicar o intervenir desde la significación vinculada al imaginario y el mundo de sentido que construyen, que operan en la diferenciación.

Estas acciones son las que construyen el mundo de sentido por medio de su designación que gatilla la realidad. Lo que se vincula con lo que en cada sistema social diferenciado se tenga por realidad, pues esto es importante, en tanto que implica no una realidad sino múltiples, por tanto para Pintos (2005, p. 43): "no hay una única realidad, que se identificaría necesariamente con una verdad única". Lo anterior por la comunicación que es funcionalmente diferenciada por los sistemas y que, por medio del sentido, incide en la construcción de la realidad y su clausura operacional.

En cuanto a su diferenciación y a la verdad, cabe agregar que el están siendo, le da un sentido diacrónico al operar de los imaginarios sociales, porque van "transformándose, adaptándose y renovándose" (Pintos, 2014, p. 7). Ante ello la importancia de la deriva histórica-cultural de la que se originan los autodescriptores.

Respecto a este operar de los imaginarios sociales, Pintos (2005, p. 44) menciona que" 1 . Los imaginarios sociales funcionan como un meta-código en los sistemas socialmente diferenciados, 2 . En el interior de un 'medio' específico (dinero, creencia, poder, etcétera) propio de cada sistema, 3. A través del código relevancia/opacidad, y 4. Generan formas y modos que fungen como realidades".

De tal modo que la comunicación es lo que estructura y dota de función al sistema social con relación al sentido y necesidades del propio sistema. De ahí que la reali- 
dad se construya desde el acoplamiento de la comunicación de cada sistema social para su mantenimiento.

Los imaginarios para Pintos (2005, p. 44) "operan en un 'medio', el medio propio de cada sistema diferenciado". Aquellos donde su significado corresponde a un fenómeno físico o simbólico reconocido, estos últimos les dan poca claridad para reconocer aquellos vinculados con imaginarios. Por ejemplo, en los sistemas funcionalmente diferenciados, el dinero es un medio con el que operan diferentes formas simbólicas socialmente generalizadas, como el crédito o las monedas, que son parte de la información con la que opera en la clausura operativa del sistema económico.

Además de operar en el medio de la diferenciación del sistema, los imaginarios sociales generan formas de naturalización de las construcciones de las realidades y la generación de modos y formas que se desempeñan como esas realidades (Pintos, 2005). Por lo tanto, pueden aportar códigos importantes para reducir la contingencia que originan las crisis.

Es en este operar donde encontramos la importancia de la propuesta teórica de los imaginarios sociales de Pintos (2005), porque al ubicarlos como esquemas socialmente construidos y meta-códigos al interior de un medio en los sistemas funcionalmente diferenciados, proporciona dos constructos muy potentes para el análisis de los imaginarios sociales que los delimitan, tanto conceptual como metodológicamente, dentro de las formas simbólicas socialmente generalizadas en la comunicación del sistema y en códigos de sentido presentes en la información, como el de relevancia/opacidad.

Pues para Pintos (2005, p. 46) las funciones de los imaginarios sociales son:"1. Producir una imagen de estabilidad en las relaciones sociales cambiantes, 2. Generar percepciones de continuidad en experiencias discontinuas, 3. Proporcionar explicaciones globales de fenómenos fragmentarios y 4. Permitir intervenir en los procesos construidos desde perspectivas diferenciadas".

La estabilidad, la continuidad, la globalidad y la diferenciación son las cuatro características funcionales de los imaginarios. Los cuales también podrían operar como unidades duales en la diferenciación funcional del sistema: estable/caótico, continuidad/discontinuo, global/local y diferenciación/homogenización, que a su vez operan en la designación de la diferenciación relevancia/opacidad. 
Entonces, el imaginario social se puede ubicar en los medios de los códigos de lo relevante y lo permanente en la comunicación funcional del sistema. Esto nos permite reducir el error conceptual de confundir los imaginarios con percepciones, representaciones o (peor aún) con la imaginación. Además de que lo relevante hace visible el sentido del sistema y la opacidad, los códigos no son precisamente necesarios para su mantenimiento, como serían los que interpenetran desde el sistema político en algunos lugares del turismo definidos como tales a partir de un programa de gobierno y no de su vocación turística, como son los PM.

De tal forma que la "nueva realidad" a la que se enfrentan los PM, sea producto de la inoperatividad de los códigos del sistema político y las exigencias estructurales para el mantenimiento del sistema económico, los cuales, en lugar de ser elementos del entorno en la comunidad, son parte de sus elementos de diferenciación para llevar a cabo su clausura operacional como un subsistema turístico, que se encuentra en crisis por la resonancia producida por la pérdida del fondo gubernamental y la pandemia del COVID-19.

No obstante, partiendo de la idea de que en la realidad se ubican esquemas en los medios por los que es simbolizada y socialmente generalizada, así como que "El mecanismo básico de construcción de esas realidades son los imaginarios sociales que nos permiten percibir algo como real a través de la articulación del código relevancia/opacidad" (Pintos, 2014, p. 6), es posible resignificar el sentido de los PM a partir de códigos que surjan de los autodefinidores de la comunidad vinculados a un imaginario del lugar, clausurando el sistema en función de sus necesidades y no de la de otros sistemas. Pero hay que tener en cuenta que los imaginarios:

No sustituyen a la realidad, sino que nos orientan en los complejos procesos por los que esta es construida como múltiple. El código básico que dirige este proceso es la distinción "relevancia/opacidad" que procede, en los procesos sociales comunicativos, a señalar como relevantes determinadas acciones, imágenes, ideas y a dejar fuera del campo de percepción otras acciones, imágenes, ideas. Se construyen así las diferentes realidades que serán válidas para los diferentes grupos según se sitúen en una o varias perspectivas. La pluralidad de experiencias, creencias, posiciones económicas y políticas y asunción de distintas identidades sociales impide que se pueda producir un denominado "pen- 
samiento único" en nuestras sociedades de acelerados cambios tecnológicos y vitales. (Pintos, 2016, p. 10).

De tal manera, el reto al que se enfrentan las comunidades de los PM es la diferenciación relevancia/opacidad, enviar los códigos que provienen del sistema político y con ello tener una reentrada de información que permita recodificar las necesidades de mantenimiento de sistema-comunidad desde su autorreferencia, ya no a partir de las necesidades estructurales de otros.

Así, se podría reducir la contingencia y delimitar la resonancia producto de las crisis económica y sanitaria. Para esto es necesario que la construcción de la realidad emerja desde sus imaginarios sociales y no de los imaginarios que se tienen sobre el turismo por parte de políticos o extranjeros. Los PM se tienen que resignificar desde su propio imaginario si quieren aminorar los efectos que a nivel de operación del subsistema turístico tienen las crisis económicas, sanitarias y, tal vez, las políticas.

\section{Conclusiones}

Si la resonancia es una propiedad del sistema con la capacidad estructural para producir irritaciones y expresar cambios cualitativos en su acoplamiento, esta puede favorecer la transformación del sistema en función de un sentido que abarque las necesidades de los habitantes y no la de otros sistemas como lo son el económico y político; cabe decir que se expresa desde adentro del sistema hacia afuera, como una expresión propia del sentido y no desde afuera hacia adentro como una imposición de un sentido cosmético.

Lo anterior por medio de los imaginarios que al ser esquemas socialmente construidos permiten la construcción de la realidad. Con ello se podría reconfigurar el sentido de los PM reduciendo su contingencia producto. Un ejemplo de ello lo podemos ubicar en lo dicho por Eloy Méndez $(2017$, p. 182) respecto a las narrativas del PPM frente a la de los habitantes, puesto que:

Se fundamenta una narrativa de los pueblos turísticos «mágicos» de México, donde barrios y pueblos son todo lugar. Desplegarlos, describirlos y descifrarlos implica rescatar las historias que de ellos se platican. Es partir de y regresar a unidades territoriales de consistencia material sujeta a modifica- 
ciones y percepción cambiante en el devenir, es hablar de la cara tangible de universos físicos, según relaciones intangibles retenidas en la memoria y la imaginación.

Donde la importancia de rescatar esas narrativas vinculadas a un sentido de lugar de los habitantes permite construir un sentido de comunidad, desde sus propios códigos y autodescriptores, con lo cual se podrían reducir los efectos negativos de la resonancia producto de las crisis económicas y sanitarias, pues el efecto negativo de estas se da en función de las necesidades estructurales del sistema político y económico. No se quiere decir con esto que no repercuta en la comunidad, pero pueden acoplarse en la comunicación para transformar el sentido del PM como programa de gobierno a un sentido de lugares comunitarios con vocación turística, desde los códigos y necesidades estructurales de la comunidad como subsistema social.

\section{Referencias}

Aragón, M. (2011). La teoría de sistemas autopoiéticos. Una breve introducción a una teoría radical de la sociedad. Revista Sociología y Tecnociencia, 2(1), 1-15. https://bit.ly/2Zq7HVo

Aragón, M. (2015). El colonial pop y lo imaginario de la mexicanidad en los Pueblos Mágicos. En I. Rodríguez-Chumillas, A. Rodríguez de Castro, G. Rodríguez-Chumillas y C. Cabrerizo (Dirs.), Entre pliegues del turismo: Desde el territorio y las representaciones en España y México. Universidad Autónoma de Madrid. http://dx.doi.org/10.15366/esmx.turismo2015

Aragón, M. (2017). El imaginario de lo sustentable como resonancia del sistema social moderno. Utopía y Praxis Latinoamericana, 22(76), 57-69. https://bit.ly/ 3dzxBhV

Aragón, M., Méndez, E. y Velázquez-García, M. A. (2017a). Turismo y patrimonio. Esbozo del "Pueblo Mágico" Álamos. Topofilia, Revista Científica de Arquitectura, Urbanismo y Territorios, 9(13). https://bit.ly/3psbpZi

Aragón, M., Covarrubias, A., Martínez, C., Méndez, E., Santos, L. y Velázquez-García, M. A. (2017b). El Álamos de las casas caídas. En C. Alvarado, A. Babini, M. GuiIlén, E. Méndez, S. Rojo y M. A. Velázquez (Coords.), Lugares de encuentro. Una visita a pueblos mexicanos. El Colegio de Hidalgo. 
Aragón, M., Balslev, H., García, A., Méndez, E. y Velázquez-García, M. A. (2016). Los portales de Álamos. Una representación del imaginario de aldea. En C. Alvarado, A. Babini, J. Baños, V. Cabrera, M. Guillén, E. Méndez, S. Rojo y P. Zacarías (Coords.), Pueblos Mágicos. Narrativas varias. Pearson, Universidad Autónoma del Estado de México y Universidad de Sonora.

Aragón, M., Balslev, H., Martínez, C., Méndez, E., Santos, L. y Velázquez-García, M. A. (2014). Álamos: imaginarios varios. En A. Valenzuela, C. Alvarado, C. Saldaña y G. Gama (Coords.), Imaginarios del paisaje y el turismo. Entre tradición y distintivos oficiales (pp. 27-46). Juan Pablo Editores y Universidad Autónoma del Estado de México. https://bit.ly/3uUR5DQ

Beuchot, M. (2009). Tratado de hermenéutica analógica. Hacia un nuevo modelo de interpretación. Universidad Nacional Autónoma de México.

Luhmann, N. (1996a). La ciencia de la sociedad. Anthropos, Universidad Iberoamericana e Instituto Tecnológico de Estudios Superiores de Occidente.

Luhmann, N. (1996b). ¿Puede la sociedad moderna evitar los peligros ecológicos? Argumentos, 24, 7-18. https://bit.ly/3baif0i

Luhmann, N. (2002) Introducción a la teoría de sistemas. Instituto Tecnológico de Estudios Superiores de Occidente.

Luhmann, N. (2007). La sociedad de la sociedad. Herder y Universidad Iberoamericana.

Luhmann, N. (2020). Comunicación ecológica ¿Puede la sociedad moderna responder a los peligros ecológicos? Universidad Iberoamericana.

Méndez, E. (2017). Narrar la ciudad. Benemérita Universidad Autónoma de Puebla.

Pintos, J. L. (2005). Comunicación, construcción de la realidad e imaginarios sociales. Utopía y Praxis Latinoamericana, 10(29), 37-65. https://bit.ly/3u5oLxX

Pintos, J. L. (2014). Algunas apreciaciones sobre el concepto de imaginarios sociales. Revista Latina de Sociología, 4, 1-11. https://bit.ly/2MiCOug

Pintos, J. L. (2016). Las preguntas por el cambio: Los imaginarios sociales de los cambios en las referencias básicas. Eduser, Revista de educação 8(2), 1-25. https://bit.ly/2OMMrXT

Velázquez-García, M. A. y Aragón, M. (2015). Todos Santos, Baja California Sur, la experiencia de lo mexicano-americano: las misiones y el Hotel California. En L. 
López-Levi, C. Malverde y M. Figueroa (Coords.), Pueblos Mágicos. Una visión interdisciplinaria (Vol. 2). Universidad Autónoma Metropolitana Xochimilco y Universidad Nacional Autónoma de México.

Velázquez-García, M. A. y Lara-Figueroa, H. (2017). El fomento y la promoción del sistema turístico en México. El Colegio de Hidalgo. https://bit.ly/3aKi4K1 